\title{
GLOBAL APPROXIMATION IN HARMONIC SPACES
}

\author{
STEPHEN J. GARDINER, MYRON GOLDSTEIN, AND KOHUR GOWRISANKARAN
}

(Communicated by Eric Bedford)

\begin{abstract}
This paper characterizes, in terms of thinness, compact sets $K$ in a suitable harmonic space $\Omega$ which have the following property: functions which are harmonic (resp. continuous and superharmonic) on a neighbourhood of $K$ can be uniformly approximated on $K$ by functions which are harmonic (resp. continuous and superharmonic) on $\Omega$. The corresponding problems of approximating functions which are continuous on $K$ and harmonic (resp. superharmonic) on the interior $\stackrel{\circ}{K}$ are also solved.
\end{abstract}

\section{INTRODUCTION}

The theorems in this paper are inspired by the following celebrated results from the theory of uniform holomorphic approximation. We refer to [Run] and [Con, pp. 198, 201] for Runge's Theorem and to [Mer] or [Rud, Chapter 20] for Mergelyan's Theorem.

Runge's Theorem. Let $\Omega$ be an open subset of $\mathbb{C}$ and $K$ a compact subset of $\Omega$. The following are equivalent:

(a) For every function $f$ which is holomorphic on some neighbourhood of $K$ and every positive number $\varepsilon$, there is a holomorphic function $g$ on $\Omega$ such that $|g-f|<\varepsilon$ on $K$.

(b) There are no components of $\Omega \backslash K$ which are relatively compact in $\Omega$.

Mergelyan's Theorem. Let $K$ be a compact subset of $\mathbb{C}$. The following are equivalent:

(a) For every function $f$ which is continuous on $K$ and holomorphic on $\stackrel{\circ}{K}$ and every positive number $\varepsilon$, there is a complex polynomial $g$ such that $|g-f|<$ $\varepsilon$ on $K$.

(b) $\mathbb{C} \backslash K$ is connected.

In this paper we solve the corresponding approximation problems for solutions and continuous supersolutions of a large class of second-order elliptic

Received by the editors December 21, 1992; presented at the NATO Advanced Research Workshop on Classical and Modern Potential Theory and Related Fields, at Chateau Bonas, France, July 25-30, 1993.

1991 Mathematics Subject Classification. Primary 31D05; Secondary 31B35, 41A30.

The third author was supported by an NSERC grant. 
p.d.e.'s; in the case of Laplace's equation, our results are contained in recent work of the first author [Gar]. To be more specific, consider the following differential operator defined on a connected open subset $\Omega$ of $\mathbb{R}^{n}$ :

$$
L=\sum_{i} \sum_{j} a_{i j} \frac{\partial^{2}}{\partial x_{i} \partial x_{j}}+\sum_{j} b_{j} \frac{\partial}{\partial x_{j}}+c .
$$

We assume that $a_{i j}=a_{j i}$ and $c \leq 0$, that $a_{i j} \in C^{2,1}$, that $b_{j} \in C^{1,1}$, and that $c \in C^{0,1}$, where $C^{k, 1}$ denotes the class of functions on $\Omega$ which are $k$ times continuously differentiable, the $k$ th-order partial derivatives being locally Lipschitz. Further, we assume that the quadratic form associated with $L$ is positive definite on $\Omega$. The $C^{2}$ solutions of the equation $L u=0$ will be called $L$-harmonic, and the lower semicontinuous solutions of $L u \leq 0$ (in the sense of distributions) will be called L-superharmonic. We assume that there is a positive $L$-superharmonic function on $\Omega$ which is not actually $L$-harmonic.

If $A \subseteq \Omega$, we write $C(A)$ for the collection of continuous real-valued functions on $A, \mathscr{H}(A)$ for the collection of functions which are $L$-harmonic on an open set containing $A$, and $\mathscr{S}_{C}(A)$ for the collection of functions which are continuous and $L$-superharmonic on an open set containing $A$. Let $K$ be a compact subset of $\Omega$. We call $(\Omega, K)$ a Runge pair for $L$-harmonic (resp. continuous L-superharmonic) functions if, for each $u$ in $\mathscr{H}(K)$ (resp. $\mathscr{S}_{C}(K)$ ) and each $\varepsilon>0$, there exists $v$ in $\mathscr{H}(\Omega)$ (resp. $\mathscr{S}_{C}(\Omega)$ ) such that $|v-u|<\varepsilon$ on $K$. Similarly, we call $(\Omega, K)$ a Mergelyan pair for L-harmonic (resp. continuous L-superharmonic) functions if, for each $u$ in $\mathscr{C}(K) \cap \mathscr{H}(\stackrel{\circ}{K})$ (resp. $C(K) \cap \mathscr{S}_{C}(\stackrel{\circ}{K})$ ) and each $\varepsilon>0$, there exists $v$ in $\mathscr{H}(\Omega)\left(\right.$ resp. $\mathscr{S}_{C}(\Omega)$ ) such that $|v-u|<\varepsilon$ on $K$. Thus, in each case, we are approximating by globally defined functions. Finally, we define $\widehat{K}$ to be the union of $K$ with all components of $\Omega \backslash K$ which are relatively compact in $\Omega$.

In this paper we show that $(\Omega, K)$ is a Runge pair for $L$-harmonic functions if and only if $\Omega \backslash \widehat{K}$ and $\Omega \backslash K$ are $L$-thin at the same points of $K$. We also show that $(\Omega, K)$ is a Mergelyan pair for $L$-harmonic functions if and only if $\Omega \backslash \widehat{K}$ and $\Omega \backslash \stackrel{\circ}{K}$ are $L$-thin at the same points of $K$. Further, these assertions remain true if " $L$-harmonic" is replaced by "continuous $L$-superharmonic" in each case. We remark that $L$-thinness is identical to classical thinness (i.e., for Laplace's equation) for many elliptic p.d.e.'s (see [He1, Chapter VII; He2]).

In fact, we shall prove our results in the more general context of Brelot harmonic spaces, so they also deal with approximation by solutions of p.d.e.'s on Riemannian manifolds (cf. [BG]). The results concerning superharmonic approximation require fewer assumptions, so we deal with them first. For the applicability of our results to the potential theory associated with the operator $L$ described above, see [He1, Chapter VII; Pra].

\section{SUPERHARMONIC APPROXIMATION}

2.1. Throughout $\S 2, \Omega$ will be a locally compact, locally connected, connected topological space with a countable base for open sets. We suppose that there is a sheaf of continuous functions satisfying Axioms 1, 2, 3 of Brelot. (For details of Brelot harmonic spaces, see [Bre, $\mathrm{CC}, \mathrm{He} 1]$.) We assume further that $\Omega$ has a base of determining domains (i.e., Axiom $D$ ), that the function 
1 is superharmonic, and that there is a positive potential on $\Omega$. We define $\widehat{K}, \mathscr{H}(A), \mathscr{S}_{C}(A)$, Runge pairs, and Mergelyan pairs as in $\S 1$ (without the prefix " $L-")$.

Theorem 1. Let $K$ be a compact subset of $\Omega$. Then $(\Omega, K)$ is a Runge pair for continuous superharmonic functions if and only if $\Omega \backslash \widehat{K}$ and $\Omega \backslash K$ are thin at the same points of $K$.

Theorem 2. Let $K$ be a compact subset of $\Omega$. Then $(\Omega, K)$ is a Mergelyan pair for continuous superharmonic functions if and only if $\Omega \backslash \widehat{K}$ and $\Omega \backslash \stackrel{\circ}{K}$ are thin at the same points of $K$.

2.2. This section contains some preliminary lemmas. We assume throughout that $K$ is compact and define $W=\widehat{K} \backslash K$. A set $A$ in $\Omega$ will be called $\Omega$ bounded if $\bar{A}$ is a compact subset of $\Omega$. We note that our hypotheses on $\Omega$ imply that this space is metrizable. The proof of the first lemma is reproduced from [Mal, Lemma 1] for the reader's convenience.

Lemma 1. (i) If $\omega$ is an open set which contains $K$, then there are only finitely many $\Omega$-bounded connected components of $\Omega \backslash K$ which intersect $\Omega \backslash \omega$.

(ii) The set $\widehat{K}$ is compact.

To prove this, we first note that $\widehat{K}$ is closed, since $\Omega \backslash \widehat{K}$ is the union of those (open) connected components of $\Omega \backslash K$ which are not $\Omega$-bounded. In (i) we may assume that $\omega$ is $\Omega$-bounded, so $\partial \omega \cap \widehat{K}$ is compact. The latter set therefore can be covered by finitely many $\Omega$-bounded components of $\Omega \backslash K$. The remaining (disjoint) $\Omega$-bounded components of $\Omega \backslash K$ must be contained in $\omega$, so (i) is proved. Given any open cover of $\widehat{K}$, we can extract a finite subcollection of open sets whose union $V$ contains $K$. It follows from (i) that $\widehat{K} \backslash V$ is compact, so we can obtain a finite subcover of $\widehat{K}$, as required.

Lemma 2. If $\omega$ is an open set which contains $\widehat{K}$, then there is a compact set $C$ such that $\widehat{K} \subset \stackrel{\circ}{C}$ and $\widehat{C} \subset \omega$.

To prove this, let $L$ be a compact set such that $\widehat{K} \subset \stackrel{\circ}{L}$ and $L \subset \omega$. It follows from Lemma 1 that there are only finitely many $\Omega$-bounded connected components $\delta_{1}, \ldots, \delta_{l}$ of $\Omega \backslash L$ which intersect $\Omega \backslash \omega$. We fix $j$ in $\{1, \ldots, l\}$, choose $x_{j}$ in $\delta_{j} \backslash \omega$, and choose $y_{j}$ to be a point in $\Omega \backslash \widehat{L}$ which lies in the connected component $U_{j}$ of $\Omega \backslash \widehat{K}$ which contains $x_{j}$. We make an arbitrary choice of metric for $\Omega$ and define $T_{k}$ to be the connected component of $\{x \in \Omega: \operatorname{dist}(x, \widehat{K})>1 / k\}$ which contains $x_{j}$. (This exists for all large $k$.) Then $\bigcup_{k} T_{k}=U_{j}$, so $y_{j} \in T_{k}$ for all sufficiently large $k$. Hence choosing $k^{\prime}$ sufficiently large and defining

$$
C=\left\{x \in \Omega: \operatorname{dist}(x, \widehat{K}) \leq 1 / k^{\prime}\right\},
$$

we obtain a compact set which satisfies $\widehat{K} \subset \stackrel{\circ}{C}$ and $\widehat{C} \subset \omega$.

Lemma 3. If $\Omega \backslash \widehat{K}$ and $\Omega \backslash K$ are thin at the same points of $K$, then $W$ is regular for the Dirichlet problem.

To establish regularity we must show that, for each $x$ in $\partial W$, the set $\Omega \backslash W$ is not thin at $x$. Suppose that $\Omega \backslash W$ is thin at $x$. Then $\Omega \backslash \widehat{K}$ is thin at 
$x$, and, by hypothesis, $\Omega \backslash K$ must also be thin at $x$. Hence $W$, which is a subset of $\Omega \backslash K$, is thin at $x$. This leads to the contradictory conclusion that $\Omega$, being the union of $W$ and $\Omega \backslash W$, is thin at $x$. Thus the lemma is proved.

Lemma 4. If $\Omega \backslash \widehat{K}$ and $\Omega \backslash K$ are thin at the same points of $K$, then $\partial W \subseteq \partial \widehat{K}$.

To see this, let $A=\partial W \backslash \partial \widehat{K}$. Then $A$ is a relatively open subset of $\partial W$. Since $\bar{W} \subset \widehat{K}$, we conclude that $A$ is a subset of the interior of $\widehat{K}$. Hence $\Omega \backslash \widehat{K}$ is thin at all points of $A$. By hypothesis, $\Omega \backslash K$ is thin at all points of $A$, and the same must be true of $W$, which is a subset of $\Omega \backslash K$. Axiom D implies that $A$ has harmonic measure zero for each component of $W$ (see [He1, Chapter V]). It follows that each point of $A$ is irregular for the Dirichlet problem on $W$. Lemma 3 now shows that $A=\varnothing$, as required.

2.3. We turn now to the proof of Theorem 1. Suppose that $\Omega \backslash \widehat{K}$ and $\Omega \backslash K$ are thin at the same points of $K$, let $u$ be continuous and superharmonic on an open set $\omega$ which contains $K$, and let $\varepsilon>0$. We denote by $\delta_{1}, \ldots, \delta_{l}$ the $\Omega$-bounded connected components of $\Omega \backslash K$ which intersect $\Omega \backslash \omega$ (see Lemma 1). (If there are no such components, we proceed immediately to the penultimate paragraph of this section.)

We temporarily fix $j$ in $\{1, \ldots, l\}$. There is a continuous positive potential $w_{j}$ on $\Omega$ which has its support $S_{j}$ contained in $\delta_{j}$. We define

$$
w_{j}^{\prime}=w_{j}-H_{w_{j}}^{\delta_{j}} \text { on } \delta_{j},
$$

where $H_{w}^{V}$ denotes the solution to the Dirichlet problem on $V$ with boundary data $w$. The function $w_{j}^{\prime}$ is positive, continuous, and superharmonic on $\delta_{j}$ and continuously vanishes at $\partial \delta_{j}$ by Lemma 3 . Further, $w_{j}^{\prime}$ is harmonic on $\delta_{j} \backslash S_{j}$. Now let $U_{j}$ be a $\delta_{j}$-bounded open set which contains the compact sets $S_{j}$ and $\delta_{j} \backslash \omega$. We define

$$
a=1+\sup [\{u(x): x \in K\} \cup\{0\}]
$$

and

$$
s_{j}(x)=a-b_{j} w_{j}^{\prime}(x) \quad\left(x \in \delta_{j}\right),
$$

where $b_{j}$ is a positive number chosen sufficiently large to ensure that

$$
a-b_{j} w_{j}^{\prime}(x)<u(x) \quad\left(x \in \partial U_{j}\right) .
$$

Thus if we define

$$
u_{j}(x)= \begin{cases}s_{j}(x) & \left(x \in U_{j}\right), \\ \min \left\{s_{j}(x), u(x)\right\} & \left(x \in \delta_{j} \backslash U_{j}\right),\end{cases}
$$

we see that $u_{j}$ is continuous and superharmonic on $\delta_{j} \backslash S_{j}$ and that $u_{j}=u$ at all points of $\delta_{j}$ which are sufficiently close to $K$.

Having carried out the above constructions for each $j$ in $\{1, \ldots, l\}$ we can define

$$
\phi(x)= \begin{cases}u_{j}(x) & \left(x \in \delta_{j} ; j=1, \ldots, l\right), \\ u(x) & \left(x \in \omega \backslash \bigcup_{j} \delta_{j}\right) .\end{cases}
$$

This function $\phi$ is continuous and superharmonic on $(\omega \cup \widehat{K}) \backslash \bigcup_{j} S_{j}$, and the function $\phi(x)+\sum_{j=1}^{l} b_{j} w_{j}(x)$ is superharmonic on $\omega \cup \widehat{K}$. 
We again temporarily fix $j$ in $\{1, \ldots, l\}$ and define

$$
g_{j}(x)=w_{j}(x)-R_{w_{j}}^{\Omega \backslash \widehat{K}}(x) \quad(x \in \Omega) .
$$

Then $g_{j}$ is a nonnegative superharmonic function on $\delta_{j}$, and its greatest harmonic minorant on $\delta_{j}$ is a bounded harmonic function. However, $g_{j}$ is also a nonnegative subharmonic (and thus upper semicontinuous) function on $\Omega \backslash S_{j}$ which vanishes on

$$
\left\{x \in \partial \delta_{j}: \Omega \backslash \widehat{K} \text { is not thin at } x\right\}
$$

and hence has limit 0 at all points of this set. If $x \in \partial \delta_{j}$ and $\Omega \backslash \widehat{K}$ is thin at $x$, then since $\partial \delta_{j} \subseteq K$, it follows by hypothesis that $\Omega \backslash K$ is thin at $x$. Thus $\delta_{j}$, being a subset of $\Omega \backslash K$, is also thin at $x$. The set of all such points $x$ must therefore, by Axiom $\mathrm{D}$, form a set of zero harmonic measure for the Dirichlet problem on $\delta_{j}$. It follows that the greatest harmonic minorant of $g_{j}$ in $\delta_{j}$ has limit 0 almost everywhere on $\partial \delta_{j}$ with respect to harmonic measure for $\delta_{j}$ and so is identically 0 . Thus $g_{j}$ is a potential on $\delta_{j}$, so the greatest harmonic minorant of $w_{j}$ in $\delta_{j}$ is given by both

$$
R_{w_{j}}^{\Omega \backslash \widehat{K}} \text { and } H_{w_{j}}^{\delta_{j}} .
$$

Hence

$$
g_{j}=w_{j}-H_{w_{j}}^{\delta_{j}} \text { on } \delta_{j},
$$

which implies that $g_{j}$ continuously vanishes on $\partial \delta_{j}$. We now define

$$
c_{j}=\inf \left\{g_{j}(x): x \in S_{j}\right\}, \quad A_{j}=\left\{x \in \delta_{j}: g_{j}(x) \geq \min \left\{\varepsilon /\left(2 l b_{j}\right), c_{j} / 2\right\}\right\},
$$

and

$$
W_{p}=\{x \in \Omega: \operatorname{dist}(x, \widehat{K})<1 / p\} \quad(p \geq 1) .
$$

(We consider only those values of $p$ large enough to ensure that $W_{p}$ is $\Omega$ bounded.) Then

$$
\widehat{R}_{w_{j}}^{\Omega \backslash W_{p}} \uparrow R_{w_{j}}^{\Omega \backslash \widehat{K}} \quad(p \rightarrow \infty) .
$$

Thus, by Dini's theorem, there exists $p^{\prime}$ such that the functions $h_{j}$ defined by

$$
h_{j}(x)=w_{j}(x)-\widehat{R}_{w_{j}}^{\Omega \backslash W_{p^{\prime}}}(x) \quad(x \in \Omega ; j=1, \ldots, l)
$$

satisfy

$$
h_{j}(x)<g_{j}(x)+\varepsilon /\left(2 l b_{j}\right) \leq \varepsilon /\left(l b_{j}\right) \quad\left(x \in \partial A_{j} ; j=1, \ldots, l\right) .
$$

For each $j$ the function $h_{j}$ is subharmonic on $\Omega \backslash A_{j}$ and vanishes outside $W_{p^{\prime}-1}$. Hence, by the maximum principle,

$$
h_{j}(x)<\varepsilon /\left(l b_{j}\right) \quad\left(x \in \Omega \backslash A_{j} ; j=1, \ldots, l\right) .
$$

We now define

$$
v^{\prime}(x)=\phi(x)+\sum_{j=1}^{l} b_{j} h_{j}(x) \quad(x \in V),
$$

where $V=(\omega \cup \widehat{K}) \cap W_{p^{\prime}}$. This $v^{\prime}$ is continuous and superharmonic on the open set $V$ (see the conclusion of the third paragraph of this section). 
It follows easily from Lemma 2 and [Hel, §7] that there is a compact set $C$ which is not thin at any of its boundary points and satisfies $\widehat{K} \subset \stackrel{\circ}{C}$ and $\widehat{C} \subset V$. Let $\omega$ be an $\Omega$-bounded open set such that $\widehat{C} \subset \omega_{1}$ and $\bar{\omega}_{1} \subset V$, and let $L$ be a compact subset of $\Omega$ such that $\bar{\omega}_{1} \subset \stackrel{\circ}{L}$. We denote the connected components of $\Omega \backslash \widehat{C}$ by $T_{1}, \ldots, T_{k}$ (there can only be finitely many of them). For each $j$ in $\{1, \ldots, k\}$ let $t_{j}$ be a continuous positive potential on $\Omega$ with compact support in $T_{j} \backslash L$ and define $t=\sum_{j} t_{j}$. Further, we define the continuous function

$$
\psi(x)=\alpha-\beta\left(t(x)-R_{t}^{\widehat{C}}(x)\right) \quad(x \in \Omega),
$$

where

$$
\alpha=1+\sup \left[\left\{v^{\prime}(x): x \in \partial C\right\} \cup\{0\}\right]
$$

and $\beta>0$. As in the construction of $u_{j}$ above, a suitably large choice of $\beta$ ensures that the function

$$
v^{\prime \prime}(x)= \begin{cases}v^{\prime}(x) & (x \in \widehat{C}), \\ \min \left\{v^{\prime}(x), \psi(x)\right\} & \left(x \in \omega_{1} \backslash \widehat{C}\right), \\ \psi(x) & \left(x \in \Omega \backslash \omega_{1}\right)\end{cases}
$$

is continuous and superharmonic on a neighbourhood of $L$ and equals $v^{\prime}$ on $K$.

Repeating the above argument for an expanding sequence of compact sets $L_{j}$ in place of $L$, where $\widehat{L}_{j}=L_{j}$ for each $j$, we obtain a continuous superharmonic function $v$ on $\Omega$ which equals $v^{\prime}$ on $K$. Further, it follows from (1) and (2) that

$$
u=\phi \leq v^{\prime}=v<\phi+\varepsilon=u+\varepsilon \text { on } K .
$$

Thus $(\Omega, K)$ is a Runge pair for continuous superharmonic functions, as required.

2.4. The converse assertion in Theorem 1 is an immediate consequence of the two lemmas established below.

Lemma 5. Let $\Omega$ be as in $\S 2.1$, let $K$ be a compact subset of $\Omega$, and suppose that $(\Omega, K)$ is a Runge pair for continuous superharmonic functions. Then, for each $u$ in $\mathscr{H}(K)$ and each $\varepsilon>0$, there exists $v$ in $\mathscr{H}(\widehat{K})$ such that $|v-u|<\varepsilon$ on $K$.

To prove this, let $u \in \mathscr{H}(K)$ and $\varepsilon>0$. By hypothesis there exists $w_{1}, w_{2}$ in $\mathscr{S}_{C}(\Omega)$ such that $u<w_{1}<u+\varepsilon$ and $-u<w_{2}<-u+\varepsilon$ on $K$. Thus $-w_{2}<w_{1}$ on $K$ and, hence, by the maximum principle, on $\widehat{K}$. In fact, by continuity, this inequality is valid on an open set $V$ which contains $\widehat{K}$. Since $w_{1}$ has a subharmonic minorant on $V$, it has a greatest harmonic minorant, $v$ say, there. Thus $-w_{2} \leq v \leq w_{1}$ on $K$, and it follows that $|v-u|<\varepsilon$ on $K$, as required.

Lemma 6. Let $\Omega$ be as in $\S 2.1$ and $K$ a compact subset of $\Omega$. Suppose further that, for each $u$ in $\mathscr{H}(K)$ and each $\varepsilon>0$, there exists $v$ in $\mathscr{H}(\widehat{K})$ such that $|v-u|<\varepsilon$ on $K$. Then $\Omega \backslash \widehat{K}$ and $\Omega \backslash K$ are thin at the same points of $K$.

To prove Lemma 6, let $\varepsilon>0$, let $z \in K$, and suppose that $\Omega \backslash K$ is not thin at $z$. Further, let $w$ be a continuous potential on $\Omega$ which characterizes 
thinness of sets. (See [He1, Chapter I] for the existence of such a potential.) There is a compact subset $C$ of $\Omega \backslash K$ such that the function $u=R_{w}^{C}$ satisfies

$$
u(z)>R_{w}^{\Omega \backslash K}(z)-\varepsilon=w(z)-\varepsilon .
$$

Since $u \in \mathscr{H}(K)$, it follows by hypothesis that there exists $v$ in $\mathscr{H}(\widehat{K})$ such that $|v-u|<\varepsilon$ on $K$. This inequality is actually valid on a neighbourhood of $K$ by continuity. For all sufficiently large $m$, the set

$$
W_{m}=\{x \in \Omega: \operatorname{dist}(x, \widehat{K})<1 / m\}
$$

is $\Omega$-bounded and $|v-u|<\varepsilon$ on $\partial W_{m}$; whence,

$$
\left|v-\widehat{R}_{u}^{\Omega \backslash W_{m}}\right|=\left|v-H_{u}^{W_{m}}\right|=\left|H_{v-u}^{W_{m}}\right|<\varepsilon \quad \text { on } W_{m} .
$$

Thus

$$
\left|\widehat{R}_{u}^{\Omega \backslash W_{m}}-u\right| \leq\left|\widehat{R}_{u}^{\Omega \backslash W_{m}}-v\right|+|v-u|<2 \varepsilon \text { on } K .
$$

Letting $m \rightarrow \infty$, it follows that

$$
\widehat{R}_{u}^{\Omega \backslash \widehat{K}} \geq u-2 \varepsilon \text { on } K .
$$

Noting that $w \geq R_{w}^{C}=u$, we can combine (3) and (4) to obtain

$$
\widehat{R}_{w}^{\Omega \backslash \widehat{K}}(z) \geq \widehat{R}_{u}^{\Omega \backslash \widehat{K}}(z)>w(z)-3 \varepsilon .
$$

Since $\varepsilon$ can be arbitrarily small, we conclude that $\widehat{R}_{w}^{\Omega \backslash \widehat{K}}(z)=w(z)$, so $\Omega \backslash \widehat{K}$ is not thin at $z$, as required.

2.5. Turning now to the proof of Theorem 2, we record the following particular case of a result of Hansen (see [Han]).

Theorem A. Let $\Omega$ be as in $\S 2.1$ and $K$ be a compact subset of $\Omega$. The following are equivalent:

(a) For each $u$ in $C(K) \cap \mathscr{S}_{C}(\stackrel{\circ}{K})$ and each $\varepsilon>0$, there exists $v$ in $\mathscr{S}_{C}(K)$ such that $|v-u|<\varepsilon$ on $K$.

(b) For each $u$ in $C(K) \cap \mathscr{H}\left(\stackrel{\circ}{K}^{\prime}\right)$ and each $\varepsilon>0$, there exists $v$ in $\mathscr{H}(K)$ such that $|v-u|<\varepsilon$ on $K$.

(c) $\Omega \backslash K$ and $\Omega \backslash \stackrel{\circ}{K}$ are thin at the same points of $K$.

Theorem 2 is an immediate consequence of Theorems 1 and A. However, there is a simpler and more direct proof of the "if" part of Theorem 2 which we now indicate.

Let $u \in C(K) \cap \mathscr{S}_{C}(\stackrel{\circ}{K})^{2}$ and $\varepsilon>0$. Since

$$
\Omega \backslash \widehat{K} \subseteq \Omega \backslash K \subseteq \Omega \backslash \stackrel{\circ}{K},
$$

we observe, by hypothesis, that $\Omega \backslash \widehat{K}$ and $\Omega \backslash K$ are thin at the same points of $K$. It follows from Lemmas 3 and 4 that, by solving the Dirichlet problem on $\widehat{K} \backslash K$, we can extend $u$ to a function $\tilde{u}$ in $C(\widehat{K}) \cap \mathscr{S}_{C}(\stackrel{\circ}{\hat{K}})$. Also, since

$$
\boldsymbol{\Omega} \backslash \widehat{K} \subseteq \Omega \backslash \stackrel{\circ}{\hat{K}} \subseteq \Omega \backslash \stackrel{\circ}{K},
$$


we see that $\Omega \backslash \widehat{K}$ and $\Omega \backslash \stackrel{\circ}{\widehat{K}}$ are thin at the same points of $K$ and hence at the same points of $\widehat{K}$. Thus, by Theorem A, there exists $w$ in $\mathscr{S}_{C}(\widehat{K})$ such that $|w-\tilde{u}|<\varepsilon$ on $\widehat{K}$, whence $|w-u|<\varepsilon$ on $K$. Arguing as in the last two paragraphs of $\S 2.3$, we can construct $v$ in $\mathscr{S}_{C}(\Omega)$ such that $v=w$ on $\widehat{K}$. Hence $|v-u|<\varepsilon$ on $K$ as required.

\section{HARMONIC APPROXIMATION}

In this section we assume, in addition to the hypotheses of $\S 2.1$, that $\Omega$ has a base of completely determining domains and that potentials (resp. adjoint potentials) with the same point support are proportional. Further, we assume axiom $\mathrm{A}^{*}$ of quasi-analyticity, i.e., any adjoint harmonic function $u$ on a connected open set $w$, which vanishes on a neighbourhood of some point of $w$, must satisfy $u \equiv 0$ on $w$. These are the hypotheses under which de la Pradelle [Pra] proved his approximation results.

Theorem 3. Let $\Omega$ be as above and $K$ a compact subset of $\Omega$. Then $(\Omega, K)$ is a Runge pair for harmonic functions if and only if $\Omega \backslash \widehat{K}$ and $\Omega \backslash K$ are thin at the same points of $K$.

Theorem 4. Let $\Omega$ be as above and $K$ a compact subset of $\Omega$. Then $(\Omega, K)$ is a Mergelyan pair for harmonic functions if and only if $\Omega \backslash \widehat{K}$ and $\Omega \backslash \stackrel{\circ}{K}$ are thin at the same points of $K$.

The "if" part of Theorem 3 follows from Theorem 1, Lemmas 5 and 2, and [Pra, Théorème 10]; the converse follows from Lemma 6. Theorem 4 follows from Theorems 3 and $A$; the "if" part can again be proved more directly, as indicated in $\S 2.5$.

\section{ACKNOWLEDGMENTS}

The first and second authors thank the Department of Mathematics and Statistics at McGill University for financial support while on sabbatical leave from their respective institutions. The second author also acknowledges support from the Université de Montréal.

\section{ADDED IN PROOF}

Professor Hansen has kindly pointed out that our superharmonic approximation results are implied by theorems in $[\mathrm{BH}]$, which were proved in a more abstract setting. However, our main result (Theorem 1) is proved by an elementary approach which seems to be of independent interest.

\section{REFERENCES}

[BG] T. Bagby and P. M. Gauthier, Uniform approximation by global harmonic functions, NATO Adv. Study Inst. Ser. C: Math. Phys. Sci., vol. 365, Kluwer Acad., Dordrecht, 1992, pp. 15-26.

[BH] J. Bliedtner and W. Hansen, Simplicial cones in potential theory II (approximation theorems), Invent. Math. 46 (1978), 255-275.

[Bre] M. Brelot, Lectures on potential theory, Tata Institute, Bombay, 1960.

[CC] C. Constantinescu and A. Cornea, Potential theory on harmonic spaces, Springer, Berlin, 1972. 
[Con] J. B. Conway, Functions of one complex variable, second edition, Springer, New York, 1978.

[Gar] S. J. Gardiner, Superharmonic extension and harmonic approximation, Ann. Inst. Fourier (Grenoble) (to appear).

[Han] W. Hansen, Harmonic and superharmonic functions on compact sets, Illinois J. Math. 29 (1985), 103-107.

[He1] R. M. Hervé, Recherches axiomatiques sur la théorie des fonctions surharmoniques et du potentiel, Ann. Inst. Fourier (Grenoble) 12 (1962), 415-571.

[He2] _- Quelques propriétés des fonctions surharmoniques associées à une équation uniformément elliptique de la forme $L u=-\sum_{i}\left(\partial / \partial x_{i}\right)\left(\sum_{j} a_{i j} \partial u / \partial x_{j}\right)=0$, Ann. Inst. Fourier (Grenoble) 15 (1965), 215-224.

[Mal] B. Malgrange, Existence et approximation des solutions des équations aux dérivées partielles et des équations de convolution, Ann. Inst. Fourier (Grenoble) 6 (1956), 271-355.

[Mer] S. N. Mergelyan, On the representation of functions by series of polynomials on closed sets, Dokl. Akad. Nauk. SSR 78 (1951), 405-408; English transl. in Amer. Math. Soc. Transl. Ser. 1, vol. 85, Amer. Math. Soc., Providence, RI, 1953.

[Pra] A. de la Pradelle, Approximation et caractère de quasi-analyticité dans la théorie axiomatique des fonctions harmoniques, Ann. Inst. Fourier (Grenoble) 17 (1967), 383-399.

[Rud] W. Rudin, Real and complex analysis, third edition, McGraw-Hill, New York, 1987.

[Run] C. Runge, Zur Theorie der eindeutigen analytischen Funktionen, Acta Math. 6 (1885), 228244.

Department of Mathematics and Statistics, McGill University, 805 Sherbrooke Street West, Montreal, Quebec, Canada H3A 2K6

Current address, S. J. Gardiner: Department of Mathematics, University College, Dublin 4, Ireland

Current address, M. Goldstein: Department of Mathematics, Arizona State University, Tempe, Arizona 85287

E-mail address, S. J. Gardiner: gardiner@irlearn.ucd.ie

E-mail address, K. GowriSankaran: gowri@math.mcgill.ca 